\title{
gs \\ Optical circular deflector with attosecond resolution for ultrashort electron beam
}

\author{
Zhen Zhang, 'Yingchao Du, and Chuanxiang Tang \\ Department of Engineering Physics, Tsinghua University, Beijing 100084, China \\ Yuantao Ding and Zhirong Huang \\ SLAC National Accelerator Laboratory, Menlo Park, California 94025, USA \\ (Received 16 January 2017; published 25 May 2017)
}

\begin{abstract}
A novel method using high-power laser as a circular deflector is proposed for the measurement of femtosecond (fs) and sub-fs electron beam. In the scheme, the electron beam interacts with a laser pulse operating in a radially polarized doughnut mode $\left(\mathrm{TEM}_{01^{*}}\right)$ in a helical undulator, generating angular kicks along the beam in two directions at the same time. The phase difference between the two angular kicks makes the beam form a ring after a propagation section with appropriate phase advance, which can reveal the current profile of the electron beam. Detailed theoretical analysis of the method and numerical results with reasonable parameters are both presented. It is shown that the temporal resolution can reach up to $\sim 100$ attosecond, which is a significant improvement for the diagnostics of ultrashort electron beam.
\end{abstract}

DOI: $10.1103 /$ PhysRevAccelBeams.20.050702

\section{INTRODUCTION}

Ultrashort electron beams with few femtosecond (fs) and sub-fs bunch length, have been generated and applied in various fields, such as beam injection studies in laserplasma wakefield accelerator (LWFA) [1] and producing ultrashort X-ray pulses in free-electron lasers (FELs) [2]. In these applications, the precise measurement of the overall bunch length and characterization of the current profile are essential for performance optimization and understanding of the physics inside, but are extremely challenging. For example, in LWFAs, the ability of precisely controlling the current profile makes it possible to optimize the beam loading and minimizing the energy spread of the injected electron bunch [3]. As for FELs, ultrashort and temporal well-defined X-ray pulses are critical and highly desired for user experiments, e.g., molecular imaging [4] and nanocrystallography [5]. At present, one of the most promising methods is to infer the x-ray temporal structure from the electron beam diagnostics $[6,7]$.

Some methods have been proposed and studied to measure the ultrashort electron beam, which can be distinguished into different classes [8]. Coherent methods use the spectrum of the light emitted from the beam by some coherent radiation process to infer the temporal profile $[9,10]$, or detect the Coulomb field traveling with the beam by electro-optic methods [11]. The unknown phase

"zhen-zhang12@mails.tsinghua.edu.cn

Published by the American Physical Society under the terms of the Creative Commons Attribution 4.0 International license. Further distribution of this work must maintain attribution to the author(s) and the published article's title, journal citation, and DOI. information in the spectrum method needs to be recovered, leaving ambiguity in the reconstructed pulse shape. In these methods, the temporal resolution depends on the spectral bandwidth and response function of the detection system. Phase space manipulation methods, including transverse deflector [12,13] and rf zero-phasing [14], map the time coordinate onto a transverse dimension and reveal the beam temporal profile directly. Their temporal resolution relies on the induced correlation strength and the transverse measurement resolution. For the deflector method, increasing the deflecting voltage and reducing the wavelength are two ways for improving the resolution [7].

With the development of laser techniques, we can streak the electron beam at optical frequencies. As the wavelength is reduced by 4 to 5 orders from rf to laser frequency, the resolution can be dramatically improved to fs, or even attosecond (as) level. It usually requires a section of undulator resonant at the laser wavelength to introduce the interaction with the electron beam. In one proposed optical streaking scheme [15], if the electron beam is located at the slope region of intensity envelop, the energy modulation grows in amplitude along the beam. When the laser power is sufficiently high, it is possible to make the energy difference between two adjacent cycles larger than the beam's intrinsic energy spread so that a clear modulation in the projected energy distribution can be observed. Since the oscillation period of the energy distribution is related to the laser wavelength, the temporal profile can be determined from the modulation. Another scheme of optical streaking is the combination of a transverse mode laser and a rf deflecting cavity [16]. The streak from the rf deflecting cavity is orthogonal with the angular modulation from the laser so that the beam distribution at different cycles can be separated. 
The usable laser wavelength is limited by the resolution of the rf deflecting cavity. It should be noted that in the both schemes above, the temporal resolution depends on the longitudinal coordinate along the beam due to the sinusoidal modulation from the laser, which may have implications for retrieval of complex temporal profiles. In addition, it is also proposed in LWFA recently to utilize the interaction between the electron and the back of the driver laser in plasma to retrieve the beam's current profile $[17,18]$.

\section{THEORETICAL ANALYSIS}

In this paper, a novel scheme using high-power laser as a circular deflector with time-independent as-level resolution is proposed for the longitudinal diagnostics of fs and sub-fs electron beam. The scheme exploits the interaction of an ultrashort electron beam with a laser operating in a higherorder transverse mode and a helical undulator resonant at the laser frequency, which is illustrated in Fig. 1. The energy and angular modulations with the linear polarized Hermite-Gaussian $\mathrm{TEM}_{10}$ laser in a planar undulator have been studied by Zholents and Zolotorev [19]. In the helical undulator interaction, we adopt the so-called doughnut mode $\mathrm{TEM}_{01^{*}}$, which is a superposition of two LaguerreGaussian $\mathrm{TEM}_{10}$ modes with $90^{\circ}$ rotation with respect to one another [20]. Different from circular polarization, the polarization vector at every position in the radially polarized beam points toward the center of the beam. The transverse intensity distribution and the polarization direction of the laser are illustrated in Fig. 2.

The laser polarization is radial and the electric field can be expressed as

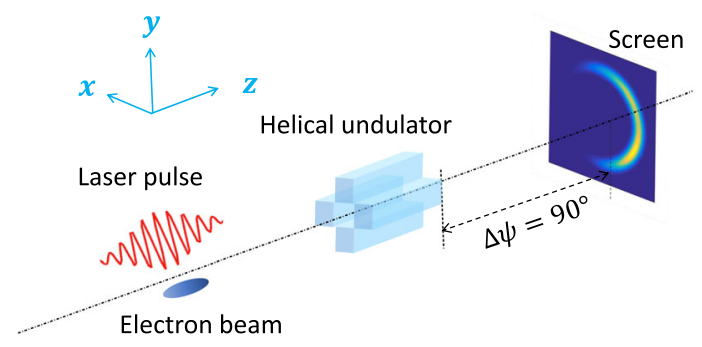

FIG. 1. Illustration of the optical circular deflector (not to scale).
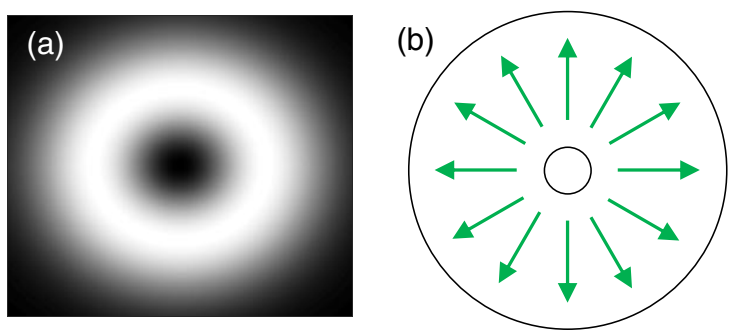

FIG. 2. (a) The transverse intensity distribution and (b) radial polarization of the doughnut mode $\mathrm{TEM}_{01^{*}}$.

$$
E_{x(y)}=\frac{E_{0}}{1+\left(z / z_{0}\right)^{2}} \frac{2 x(y)}{w_{0}} \sin (k(z-c t)+\psi) G,
$$

and the factor $G$ denotes the effects of the laser longitudinal and transverse distribution with

$$
G=e^{-\frac{(z / c-t+s / c)^{2}}{4 \sigma_{\tau}^{2}}} e^{-\frac{x^{2}+y^{2}}{w_{0}^{2}\left(1+\left(z / z_{0}\right)^{2}\right)}}
$$

Here $E_{0}$ is the field amplitude, $k=2 \pi / \lambda$ is the wave vector, $\lambda$ is the wavelength, $z_{0}=k w_{0}^{2} / 2$ is the Rayleigh length, $w_{0}$ is the waist size which is assumed to be in the center of the undulator, $\psi=\psi_{0}-2 \tan ^{-1}\left(z / z_{0}\right)+k\left(x^{2}+y^{2}\right) / 2 R, \psi_{0}=$ $k s$ is the phase of the wave at the beginning of the interaction at the entrance of the undulator, $s$ is the electron coordinate within the electron bunch, $R=\left(z^{2}+z_{0}^{2}\right) / z$, and $\sigma_{\tau}$ is the rms width of the laser pulse. For brevity, we assume that the laser pulse is long enough and the electron beam size $\sigma_{x, y} \ll w_{0}$ so that $G=1$ in the following derivations. Note that the laser and the electron motion in helical undulator are both circularly symmetric, so the definition of $x$ and $y$ directions is arbitrary here.

For electron motion inside the helical undulator, the velocity components are given by

$$
\begin{aligned}
& \beta_{x}=\frac{K}{\gamma} \cos \left(k_{u} z\right), \\
& \beta_{y}=\frac{K}{\gamma} \sin \left(k_{u} z\right),
\end{aligned}
$$

where $k_{u}=2 \pi / \lambda_{u}, \quad \lambda_{u}$ is the undulator period, $K=e B_{0} / k_{u} m c, B_{0}$ is the peak magnetic field, and $\gamma$ is the relativistic factor. Then the energy gain/loss obtained by the electron in its interaction with the laser field can be found by solving the equation

$$
\frac{d \gamma}{d t}=-\frac{e}{m c}\left(E_{x} \cdot \beta_{x}+E_{y} \cdot \beta_{y}\right) .
$$

Plugging Eqs. (1), (3) and (4), the energy change can be written as

$$
\begin{aligned}
\left\langle\frac{d \gamma}{d t}\right\rangle= & \frac{e}{m c} \frac{K E_{0}}{\gamma w_{0}\left(1+\left(z / z_{0}\right)^{2}\right)}\left[x \sin \left(k_{u} z \frac{2 \delta \gamma}{\gamma_{r}}+\phi\right)\right. \\
& \left.-y \cos \left(k_{u} z \frac{2 \delta \gamma}{\gamma_{r}}+\phi\right)\right],
\end{aligned}
$$

where the symbol $\langle\cdot\rangle$ means averaging over one undulator period. Here we also define the resonance electron energy as $\gamma_{r}^{2}=\frac{k}{2 k_{u}}\left(1+K^{2}\right)$ and $\delta \gamma$ represents the energy deviation. Taking the dimensionless variables: $\hat{z}=c t / L_{u}$, $\nu=N 2 \delta \gamma / \gamma_{r}$ and $q=L_{u} / z_{0}, q \hat{z}=z / z_{0}$, where $L_{u}=$ $N_{u} \lambda_{u}$ is the length of the undulator with $N_{u}$ periods, and replacing the field $E_{0}$ by the laser power $P_{L}=E_{0}^{2} \pi w_{0}^{2} / 2 \mu_{0} c$, the energy modulation becomes 


$$
\left\langle\frac{d \gamma}{d \hat{z}}\right\rangle=\frac{\sqrt{2} K}{\gamma} \sqrt{\frac{P_{L}}{P_{0}}} k q \frac{\left[x \sin \left(2 \pi \nu \hat{z}-2 \tan ^{-1}(q \hat{z})+k s\right)-y \cos \left(2 \pi \nu \hat{z}-2 \tan ^{-1}(q \hat{z})+k s\right)\right]}{1+(q \hat{z})^{2}}
$$

with $P_{0}=I_{A} m c^{2} / e$ and the Alfvén current $I_{A}$. By using the Panofsky-Wentzel theorem [21],

$$
\frac{\partial \Delta x^{\prime}}{\partial s}=\frac{\partial}{\partial x}\left(\frac{\Delta \gamma}{\gamma}\right), \quad \frac{\partial \Delta y^{\prime}}{\partial s}=\frac{\partial}{\partial y}\left(\frac{\Delta \gamma}{\gamma}\right),
$$

we can obtain the angular kicks due to the interaction with the laser [19]. By integrating $\hat{z}$ from -0.5 to 0.5 (over the undulator distance), the angular kicks on the electron beam after passing through the undulator can be formulated as

$$
\begin{aligned}
& \Delta x^{\prime}=-\frac{\sqrt{2} K}{\gamma^{2}} \sqrt{\frac{P_{L}}{P_{0}}} f(q, \nu) \cos (k s+\phi), \\
& \Delta y^{\prime}=-\frac{\sqrt{2} K}{\gamma^{2}} \sqrt{\frac{P_{L}}{P_{0}}} f(q, \nu) \sin (k s+\phi),
\end{aligned}
$$

where $\phi$ is an arbitrary phase and

$$
f(q, \nu)=q \int_{-0.5}^{0.5} \frac{\cos \left[2 \pi \nu \hat{z}-2 \tan ^{-1}(q \hat{z})\right]}{1+(q \hat{z})^{2}} d \hat{z} .
$$

In Eqs. (9) and (10), it is founded that there is a phase difference, $\pi / 2$, between the kicks of the two directions, leading to the relations

$$
\begin{gathered}
\Delta r^{\prime}=\sqrt{\left(\Delta x^{\prime 2}+\Delta y^{\prime 2}\right)}=\frac{\sqrt{2} K}{\gamma^{2}} \sqrt{\frac{P_{L}}{P_{0}}} f(q, \nu), \\
\theta=\arctan \left(\Delta y^{\prime} / \Delta x^{\prime}\right)=k s+\phi,
\end{gathered}
$$

which means that the kick strength along the beam is uniform (ignore the energy spread here) and the kick angle only linearly depends on the electron's longitudinal position within the bunch. It could further conclude that if the beam size is dominant by the induced kicks, then after propagation with $\pi / 2$ phase advance, the beam will form a ring on the screen (see Fig. 1). The radius of the ring is determined by the kick strength and independent of the longitudinal coordinate. The distribution along the polar angle on the screen can be used to reconstruct the beam's temporal profile, which is similar with an rf deflecting cavity, so we can call it as a circular deflector.

To consider the temporal resolution of the proposed circular deflector, we assume the beam has an initial rms angular spread $\sigma_{x^{\prime}}$ and define the normalized kick strength as

$$
\mathcal{R}=\frac{\Delta r^{\prime}}{\sigma_{x^{\prime}}}
$$

Here $\mathcal{R}$ can also represent the radius of the ring on the screen normalized by the unperturbed rms beam size after beam propagation with $\pi / 2$ phase advance. Then the resolution of the polar angle is $\sigma_{\theta}=1 / \mathcal{R}$, and according to Eq. (13), the corresponding temporal resolution of the circular deflector is

$$
\sigma_{t}=\frac{\sigma_{\theta}}{k c}=\frac{1}{k c \mathcal{R}}=\frac{1}{2 \pi \mathcal{R}} \cdot \frac{\lambda}{c},
$$

where $\lambda$ is the adopted laser wavelength. The equation above shows a great potential to achieve very high temporal resolution. For example, using $\sim 1 \mu \mathrm{m}$ wavelength laser and $\mathcal{R} \approx 10$, the temporal resolution can reach up to $\sim 50$ as.

Note that the function $f(q, \nu)$ in Eq. (11) does not include the effects of the finite pulse length and transverse size of the laser pulse. However, it is possible to generalize the expression to the case with these effects included. The general expression of $f\left(q, \nu, \sigma_{\tau}, s, w_{0}, z_{0}\right)$ can be found in Ref. [19] although it was for the interactions in a planar undulator. The degradation of $f(q, \nu)$ will reduce the kick strength for electrons, but has little effects on the beam distribution along the polar angle. We will adopt the general expression in the following numerical calculations.

\section{NUMERICAL RESULTS}

In this section, we present some numerical results and application examples of the circular deflector with reasonable parameters. The choice of the laser wavelength should be based on the requirement of temporal resolution and measurement range. Smaller wavelength makes it easier to achieve higher resolution, but also limits the available measurement range. In the following examples, we use the laser of $4 \mu \mathrm{m}$ for the circular deflector. The high-power $4 \mu \mathrm{m}$ laser can be generated by optical parametric chirped pulse amplification [22,23] from $800 \mathrm{~nm}$ laser systems, which have been widely used in modern accelerator facilities and LWFA experiments.

For the design of a circular deflector, we first need to determine the parameters of the helical undulater and the laser. We use $4 \mu \mathrm{m}$ laser and $1 \mathrm{GeV}$ electron beam as an example. The undulator period $\lambda_{u}=10.3 \mathrm{~cm}$ and the corresponding $K=17.3$. Through scanning the undulator period $N_{u}$, laser waist size $w_{0}$ and detune $\nu$, we choose the parameters $N_{u}=2, w_{0}=300 \mu \mathrm{m}$ and $\nu=0.72$, where the function $f(q, \nu)=1$.9. The maximum value of $f(q, \nu)$ can 

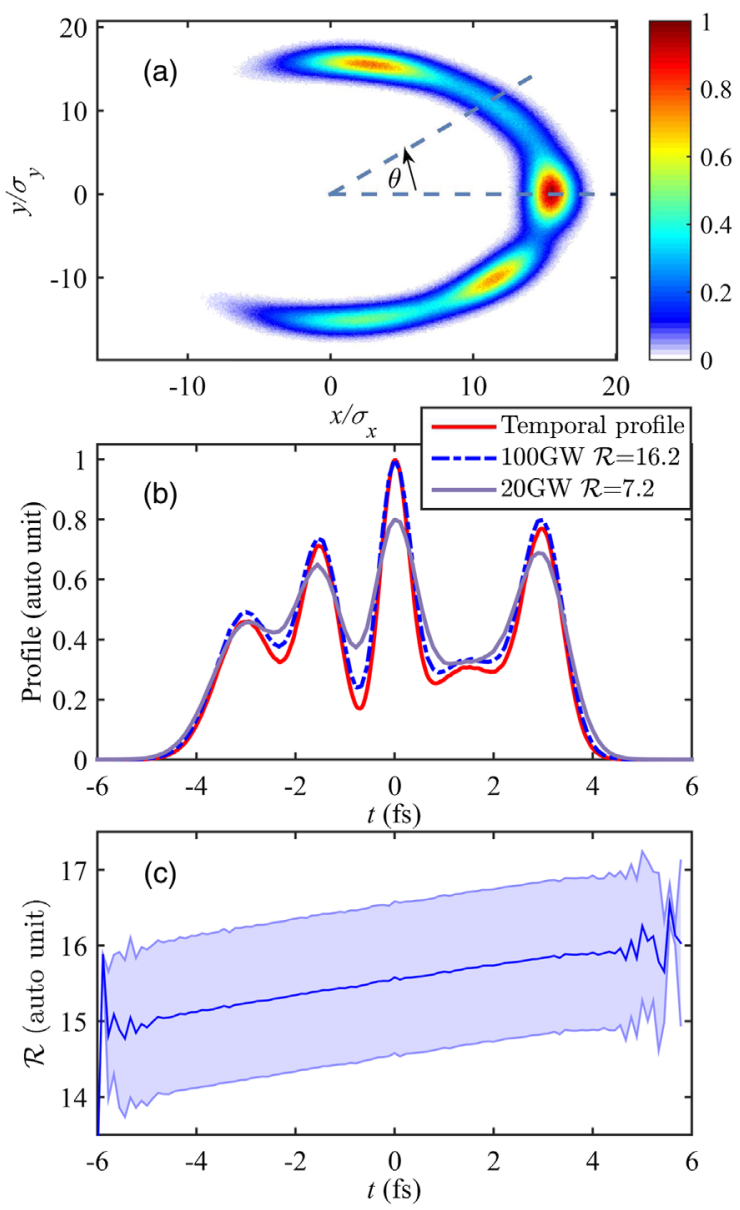

FIG. 3. Circular deflector results: (a) ring pattern at the final screen when the laser power is $100 \mathrm{GW}$; (b) actual and reconstructed beam current profiles with laser power $100 \mathrm{GW}$ and $20 \mathrm{GW}$; (c) the average ring radius along the beam time coordinate with laser power $100 \mathrm{GW}$. The shadow is the rms width of the radius.

be up to $\sim 2.4$, but in this case it requires more undulator periods and smaller laser waist size.

The electron beam parameters we adopt are as follows: the normalized emittance is $0.1 \mu \mathrm{m}$ and the full width at half maximum (FWHM) of the beam profile is $\sim 6 \mathrm{fs}$. In order to test the resolution, we add complex structures onto the current profile as shown in Fig. 3(b). There is also an energy chirp on the beam, which scales $\sim 3 \%$ over the whole bunch. These numbers above are reasonable in LWFA. The rms beam size at the deflector is $20 \mu \mathrm{m}$, which can be matched by the phase space matching technique in LWFA [24]. In this case the laser pulse length is much larger than the electron bunch length, so we can ignore the power envelop and slippages in the undulator.

Figure 3 presents the results of the circular deflector. Here we take two kinds of laser power, $100 \mathrm{GW}$ and $20 \mathrm{GW}$ for comparison. According to Eqs. (14) and (15), the temporal resolution is $\sim 130$ as for $100 \mathrm{GW}(\mathcal{R}=16.2)$ and $\sim 300$ as for $20 \mathrm{GW}(\mathcal{R}=7.2)$. The ring pattern of the
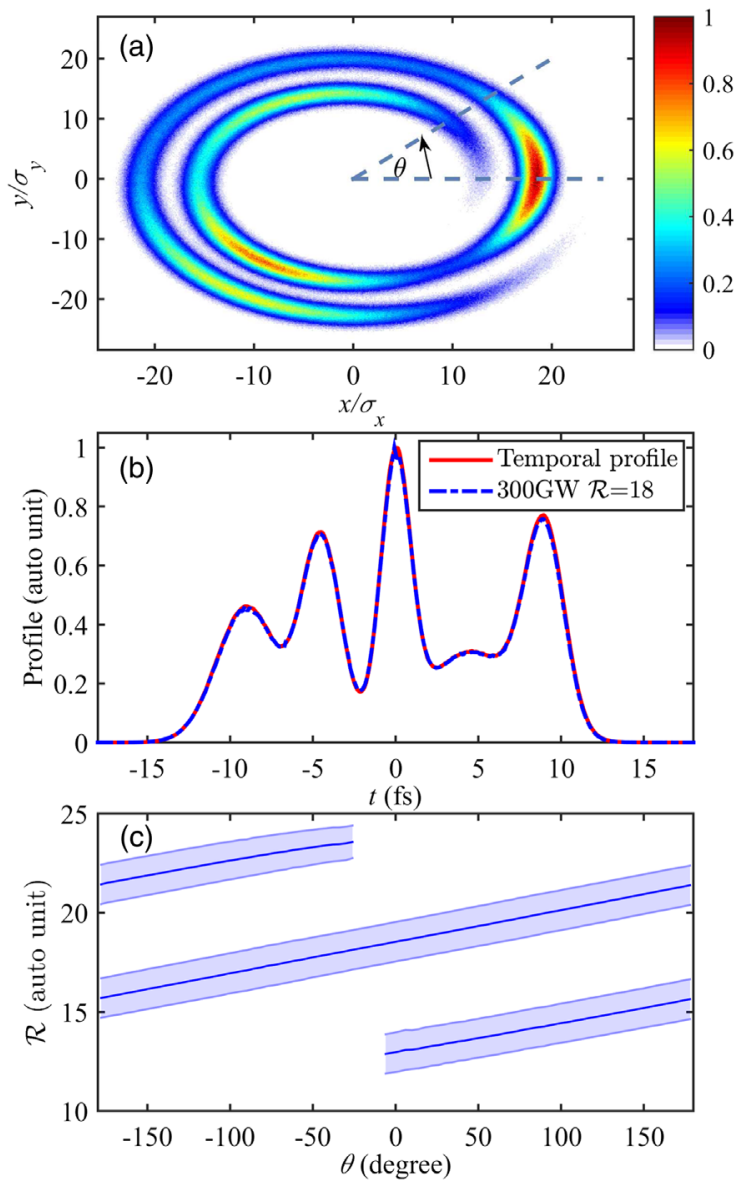

FIG. 4. Circular deflector results for longer electron beam: (a) spiral pattern at the final screen when the laser power is $300 \mathrm{GW}$; (b) actual and reconstructed beam current profiles with laser power $300 \mathrm{GW}(\mathcal{R}=18)$; (c) the average ring radius along the polar angles. The shadow is the rms width of the radius.

beam at the final screen is shown in Fig. 3(a). Projecting along the polar angle, the temporal profile can be reconstructed in Fig. 3(b). It can be seen that even at $20 \mathrm{GW}$ laser power, the reconstructed profile agrees well with the pre-set profile. Compared with the result of $100 \mathrm{GW}$ case, the contrasting structures in the profile, e.g. narrow peaks and valleys, become gentle due to the relative lower temporal resolution at $20 \mathrm{GW}$. In addition to the temporal profile, if we assume the laser power is uniform, we can also estimate the energy chirp of the beam by the average radius of the ring pattern along the polar angle. In Fig. 3(c), from the head to tail, there is $\sim 7 \%$ difference in the radius $\mathcal{R}$, which is a bit larger than the twice of the 3\% energy chirp of the beam. This is because the kick strength is proportional to the inverse square of the energy and the offset of the detune will reduce the kick strength further.

In the examples above, we limit the electron bunch length less than the adopted laser wavelength. In this case, the dynamic range of the deflector is $2 \pi \mathcal{R}$. In order to measure the bunch length which is longer than the laser 
wavelength, especially several times of the wavelength, we can synchronize the beam at the region with maximum "power slope" in the laser pulse temporal profile. Here the power slope means the longitudinal rise or drop of a Gaussian power envelop. Different parts of the electron beam are modulated with different power or electric field, and the difference in the kick strength can distinguish the adjacent rings to form a spiral pattern. Using the same current profile, we scale the time coordinates of all electrons by 3 times and the FWHM of the beam becomes $\sim 18$ fs. The laser pulse length is rms 30 fs and the laser power is increased to $300 \mathrm{GW}$ to provide enough kick difference for the adjacent rings. The electron beam is delayed by 40 fs to lie at the large slope of the intensity envelope. The other parameters of laser and undulator are kept the same with the previous cases. The results are given in Fig. 4. From the top plot (a), it can be seen clearly that the adjacent rings of the spiral are separated by the circular deflector. This can be verified from Fig. 4(c) as well, where the average radius and its rms width along the polar angle are given. The separation between the adjacent rings is $\sim 6$ times of the rms width. Expanding the spiral along the polar angle, we can reconstruct the temporal profile, as shown in Fig. 4(b). The temporal resolution in this case is 120 as $(\mathcal{R}=18)$, which is enough to reveal every detailed structures in the profile.

\section{DISCUSSIONS}

The proposed method of optical circular deflector has some advantages in beam diagnostics. Compared with the usual rf deflector, besides the higher temporal resolution, the measurement of optical circular deflector is selfcalibrated. For the rf deflector, it is necessary to do calibration, since the mapping from longitudinal coordinate to transverse position is determined by the kick strength of the deflector and the transfer matrix of the beam propagation section. However, in the optical circular deflector, the temporal profile is obtained from the polar angle distribution, where one circle $\left(360^{\circ}\right)$ corresponds to one laser wavelength. The kick strength and the transfer matrix after the deflector will change the temporal resolution, but have little effect on this calibration coefficient.

In addition, the proposed optical circular deflector method is suitable for wide range of beam energy. Here we give a scaling law of temporal resolution. If we keep the peak magnetic field of the undulator, according to the resonant equation, $K \propto \gamma^{2 / 3}$. If we further assume the beam size at the deflector and the normalized transverse emittance are kept the same, the initial angular spread $\sigma_{x^{\prime}} \propto \gamma^{-1}$. Then the scale law of temporal resolution can be written as

$$
\sigma_{t} \propto \frac{1}{\mathcal{R}} \propto \frac{K P_{L}^{1 / 2}}{\gamma^{2} \sigma_{x^{\prime}}} \propto \frac{P_{L}^{1 / 2}}{\gamma^{1 / 3}}
$$

It means that for different beam energy, if we want to keep the same temporal resolution, the laser power should be $P_{L} \propto \gamma^{2 / 3}$. In the previous examples, the beam energy is $1 \mathrm{GeV}$. If we increase it to $4 \mathrm{GeV}$, the laser power needs to be increased by a factor of 2.5 to maintain the temporal resolution, which is still very reasonable.

Lastly, we talk about the effects of intrinsic spread in beam parameters on the application of the optical circular deflector, especially when the bunch length is larger than the laser wavelength. Here we consider the beam with energy chirp $\gamma(s)=\gamma_{0}(1-a s)$, intrinsic energy spread $\sigma_{\gamma}$ and normalized emittance $\epsilon_{N}$, where $|a| \ll 1$ denotes chirp slope. Using the laser with power slope $P_{L}(s)=P_{L 0}(1+b s)$, the difference of the kick strength between the adjacent rings is

$$
\begin{aligned}
S_{\Delta r^{\prime}} & =\frac{\sqrt{2} K}{\gamma_{0}^{2}} \sqrt{\frac{P_{L 0}}{P_{0}}} f(q, \nu)\left(2 a+\frac{1}{2} b\right) \lambda, \\
& =\Delta r^{\prime}\left(2 a+\frac{1}{2} b\right) \lambda,
\end{aligned}
$$

where $\lambda$ is the laser wavelength and the linear slope is an approximation for the range of laser wavelength. Due to the intrinsic energy spread and angular divergence, the rms width of the ring or spiral can be written as

$$
\sigma_{\Delta r^{\prime}}=\sqrt{\left(2 \Delta r^{\prime} \frac{\sigma_{\gamma}}{\gamma_{0}}\right)^{2}+\left(\frac{\epsilon_{N}}{\gamma_{0} \sigma_{x}}\right)^{2}} .
$$

In order to resolve the adjacent rings, the difference of kick strength has to be several times $(>4)$ larger than the rms width,

$$
\frac{S_{\Delta r^{\prime}}}{\sigma_{\Delta r^{\prime}}}>4
$$

From the analysis above, the kick difference comes from the combination of beam energy chirp and laser power slope. Longer laser wavelength is helpful to resolve the spiral. For the beam without energy chirp $(a=0)$, we have to increase laser power and power slope to obtain enough kick difference. The rms width of the ring reduces the temporal resolution and blurs the transverse pattern. Increasing beam size at the deflector helps reduce the emittance effect. However, considering the assumption that the laser waist size is much larger than the beam size, it requires more undulator periods in this case consequently. Besides, the beam focusing mismatch and error in phase advance will also contribute to the blurring effect and reduce the temporal resolution. The qualitative analysis for these factors is similar with the one in conventional $\mathrm{rf}$ deflectors. 


\section{SUMMARY}

In this paper, a novel method for the ultrashort electron beam measurement, optical circular deflector, has been proposed. The scheme takes advantages of the interactions of the electron beam with a laser operating in doughnut mode in a helical undulator to generate uniform circular kicks in the transverse dimensions. Since the laser wavelength is much shorter than the rf wavelength, the temporal resolution can be improved up to as level. In the paper, we have presented some examples with $4 \mu \mathrm{m}$ laser with temporal resolution approaching $\sim 100$ as. The method still works for other wavelengths, for example, $800 \mathrm{~nm}$ and $10 \mu \mathrm{m}$. The choice of the laser wavelength should depend on the requirement of resolution and the bunch length range of the electron beam. The measurement range can be increased by several times through locating the electron beam at the region with maximum power slope in the laser power envelop. The proposed method is self-calibrated and it is easy to scale to higher energy. In summary, the proposed method provides a powerful tool for the diagnostics of the fs and sub-fs electron beam. It is of great potential to apply in LWFA experiments and FEL facilities, where high-power laser systems are readily available.

\section{ACKNOWLEDGMENTS}

This work was supported by the National Natural Science Foundation of China (NSFC Grants No. 11375097 and No. 11435015) and U.S. Department of Energy Contracts No. DE-AC02-76SF00515.

[1] O. Lundh, J. Lim, C. Rechatin, L. Ammoura, A. Ben-Ismail, X. Davoine, G. Gallot, J.-P. Goddet, E. Lefebvre, V. Malka et al., Few femtosecond, few kiloampere electron bunch produced by a laser-plasma accelerator, Nat. Phys. 7, 219 (2011).

[2] Y. Ding, A. Brachmann, F.-J. Decker, D. Dowell, P. Emma, J. Frisch, S. Gilevich, G. Hays, P. Hering, Z. Huang et al., Measurements and Simulations of Ultralow Emittance and Ultrashort Electron Beams in the Linac Coherent Light Source, Phys. Rev. Lett. 102, 254801 (2009).

[3] M. Tzoufras, W. Lu, F. Tsung, C. Huang, W. Mori, T. Katsouleas, J. Vieira, R. Fonseca, and L. Silva, Beam Loading in the Nonlinear Regime of Plasma-Based Acceleration, Phys. Rev. Lett. 101, 145002 (2008).

[4] N. Rohringer, D. Ryan, R. A. London, M. Purvis, F. Albert, J. Dunn, J. D. Bozek, C. Bostedt, A. Graf, R. Hill et al., Atomic inner-shell X-ray laser at 1.46 nanometres pumped by an X-ray free-electron laser, Nature (London) 481, 488 (2012).

[5] A. Barty, C. Caleman, A. Aquila, N. Timneanu, L. Lomb, T. A. White, J. Andreasson, D. Arnlund, S. Bajt, T. R. Barends et al., Self-terminating diffraction gates femtosecond X-ray nanocrystallography measurements, Nat. Photonics 6, 35 (2012).
[6] Y. Ding, C. Behrens, P. Emma, J. Frisch, Z. Huang, H. Loos, P. Krejcik, and M. Wang, Femtosecond X-ray pulse temporal characterization in free-electron lasers using a transverse deflector, Phys. Rev. ST Accel. Beams 14, 120701 (2011).

[7] C. Behrens, F.-J. Decker, Y. Ding, V. Dolgashev, J. Frisch, Z. Huang, P. Krejcik, H. Loos, A. Lutman, T. Maxwell et al., Few-femtosecond time-resolved measurements of X-ray free-electron lasers, Nat. Commun. 5 (2014).

[8] H. Loos, Longitudinal diagnostics for short electron beam bunches, in Proceedings of the 23rd Particle Accelerator Conference, Vancouver, Canada (IEEE, Piscataway, NJ, 2009).

[9] A. Debus, M. Bussmann, U. Schramm, R. Sauerbrey, C. Murphy, Z. Major, R. Hörlein, L. Veisz, K. Schmid, J. Schreiber et al., Electron Bunch Length Measurements from Laser-Accelerated Electrons Using Single-Shot THz Time-Domain Interferometry, Phys. Rev. Lett. 104, 084802 (2010).

[10] T. J. Maxwell, C. Behrens, Y. Ding, A. Fisher, J. Frisch, Z. Huang, and H. Loos, Coherent-Radiation Spectroscopy of Few-Femtosecond Electron Bunches Using a MiddleInfrared Prism Spectrometer, Phys. Rev. Lett. 111, 184801 (2013).

[11] G. Berden, S. P. Jamison, A. M. MacLeod, W. Gillespie, B. Redlich, and A. van der Meer, Electro-Optic Technique with Improved Time Resolution for Real-Time, Nondestructive, Single-Shot Measurements of Femtosecond Electron Bunch Profiles, Phys. Rev. Lett. 93, 114802 (2004).

[12] P. Emma, J. Frisch, and P. Krejcik, LCLS Technical Note LCLS-TN-00-12 (2000).

[13] D. Xiang and Y. Ding, Longitudinal-to-transverse mapping for femtosecond electron bunch length measurement, Phys. Rev. ST Accel. Beams 13, 094001 (2010).

[14] Z. Huang, K. Bane, Y. Ding, and P. Emma, Single-shot method for measuring femtosecond bunch length in linacbased free-electron lasers, Phys. Rev. ST Accel. Beams 13, 092801 (2010).

[15] Y. Ding, Z. Huang, and K. L. Bane, An optical streaking method for measuring femtosecond electron bunches, in Proceedings of the 2011 Free-electron Laser Conference, Shanghai, China (FEL'11/EPS-AG, Shanghai, 2011).

[16] G. Andonian, E. Hemsing, D. Xiang, P. Musumeci, A. Murokh, S. Tochitsky, and J. Rosenzweig, Longitudinal profile diagnostic scheme with subfemtosecond resolution for high-brightness electron beams, Phys. Rev. ST Accel. Beams 14, 072802 (2011).

[17] H. Kotaki, K. Kawase, Y. Hayashi, M. Mori, M. Kando, J. K. Koga, and S. V. Bulanovt, Direct observation of the pulse width of an ultrashort electron beam, J. Phys. Soc. Jpn. 84, 074501 (2015).

[18] C. Zhang, J. Hua, Y. Wan, B. Guo, C.-H. Pai, Y. Wu, F. Li, H.-H. Chu, Y. Gu, W. Mori et al., Temporal characterization of ultrashort linearly chirped electron bunches generated from a laser wakefield accelerator, Phys. Rev. Accel. Beams 19, 062802 (2016).

[19] A. Zholents and M. Zolotorev, Attosecond x-ray pulses produced by ultra short transverse slicing via laser electron beam interaction, New J. Phys. 10, 025005 (2008). 
[20] V. Niziev, R. Chang, and A. Nesterov, Generation of inhomogeneously polarized laser beams by use of a Sagnac interferometer, Appl. Opt. 45, 8393 (2006).

[21] W. Panofsky and W. Wenzel, Some considerations concerning the transverse deflection of charged particles in radio-frequency fields, Rev. Sci. Instrum. 27, 967 (1956).

[22] G. Andriukaitis, T. Balčiūnas, S. Ališauskas, A. Pugžlys, A. Baltuška, T. Popmintchev, M.-C. Chen, M. M. Murnane, and H. C. Kapteyn, $90 \mathrm{GW}$ peak power fewcycle mid-infrared pulses from an optical parametric amplifier, Opt. Lett. 36, 2755 (2011).
[23] K. Zhao, H. Zhong, P. Yuan, G. Xie, J. Wang, J. Ma, and L. Qian, Generation of $120 \mathrm{GW}$ mid-infrared pulses from a widely tunable noncollinear optical parametric amplifier, Opt. Lett. 38, 2159 (2013).

[24] X. Xu, J. Hua, Y. Wu, C. Zhang, F. Li, Y. Wan, C.-H. Pai, W. Lu, W. An, P. Yu et al., Physics of Phase Space Matching for Staging Plasma and Traditional Accelerator Components Using Longitudinally Tailored Plasma Profiles, Phys. Rev. Lett. 116, 124801 (2016). 